\title{
Sin dejar a nadie atrás: una metodología para establecer metas de reducción de desigualdad en salud del Objetivo de Desarrollo Sostenible 3
}

\author{
Antonio Sanhueza ${ }^{1}$, Isabel Espinosa', Oscar J. Mújica' y Jarbas Barbosa da Silva Jr. ${ }^{1}$
}

Forma de citar

Sanhueza A, Espinosa I, Mújica OJ, da Silva Jr. JB. Sin dejar a nadie atrás: una metodología para establecer metas de reducción de desigualdad en salud del Objetivo de Desarrollo Sostenible 3. Rev Panam Salud Publica. 2020;44:e155. https:// doi.org/10.26633/RPSP.2020.155

RESUMEN

Objetivos. Presentar una metodología para la formulación simultánea de metas cuantitativas que reflejen tanto la mejoría del promedio nacional de un indicador del tercer Objetivo de Desarrollo Sostenible (ODS3) como la reducción de su desigualdad geográfica.

Métodos. Se definió un algoritmo en cinco pasos: a) cálculo del cambio porcentual anual promedio (CPAP) nacional para un indicador del ODS3; b) definición normativa de estratos geográficos a partir de la distribución subnacional del indicador en un año base; c) aplicación de un criterio de progresividad proporcional del CPAP para proyectar el indicador estrato-específico al año meta; d) establecimiento de la meta nacional como el promedio ponderado del indicador en las unidades territoriales subnacionales al año meta; y e) formulación de metas de reducción de desigualdad mediante el cálculo de las brechas absoluta y relativa entre los estratos extremos al año meta.

Resultados. Se aplicó el algoritmo al indicador ODS 3.1.1 (razón de mortalidad materna, RMM), desagregado por los 22 departamentos de Guatemala para el año base 2014 (RMM = 113 por 100000 nacidos vivos). Sosteniendo la intensidad promedio de CPAP observada entre 2009 y 2014 (-4,3\%) y focalizando sus acciones con progresividad territorial, el país reduciría al 2030 su RMM a 53 por 100000 nacidos vivos y sus brechas absoluta y relativa en $72 \%$ y $48 \%$, respectivamente.

Conclusiones. La metodología propuesta permite formular simultáneamente metas de reducción de las desigualdades geográficas en salud y hacer explícita la primacía del principio de equidad expresado en el compromiso de no dejar a nadie atrás que identifica a los ODS, cuya urgencia cobra renovada relevancia en el escenario pospandémico actual.

Palabras clave Desarrollo sostenible; equidad en salud; indicadores de salud; mortalidad materna; Guatemala.

Desde septiembre de 2015, el mundo cuenta con una nueva agenda global para transformar la vida de las personas y su entorno natural, conocida como la Agenda 2030 de Desarrollo Sostenible y definida como un plan de acción con 17 objetivos en favor de la prosperidad y la paz mundiales (1). Estos denominados Objetivos de Desarrollo Sostenible (ODS) se consideran indivisibles y son más exhaustivos y ambiciosos que sus predecesores, los Objetivos de Desarrollo del Milenio (ODM). Por ejemplo, a diferencia de estos últimos, los ODS van más allá de los temas tradicionales de la pobreza e incluyen objetivos relacionados con la paz, los derechos humanos y la buena gobernanza, como trazadores del progreso. Los ODS se organizan en un marco común de 169 metas y 244 indicadores mundiales (2), el tercero de esos objetivos (ODS3) está enfocado en "garantizar una vida sana y promover el bienestar para todos en todas las edades", a cuyo alcance se aspira bajo la promesa de no dejar a nadie atrás (1).

\footnotetext{
1 Organización Panamericana de la Salud; Washington, DC., Estados Unidos de América. $\bowtie$ Antonio Sanhueza, sanhueza@paho.org
} 
En contraste con este tácito reconocimiento a la universalidad y la equidad social como elementos centrales en la construcción de oportunidades al desarrollo sostenible, las metas de los ODS con vistas al año 2030 se han formulado de forma análoga a los ODM, ya que consideran únicamente cambios explícitos en los promedios nacionales y globales de sus indicadores, sin tomar en cuenta la desigualdad distributiva al interior de los países. Por ejemplo, la primera meta del ODS3 (meta 3.1) propone: "De aquí al 2030, reducir la tasa mundial [sic] de mortalidad materna a menos de 70 por cada 100000 nacidos vivos" (2). Tal formulación, basada en un valor promedio a nivel mundial, resulta insuficiente para rastrear la evolución de la desigualdad (en este caso, en el riesgo de muerte materna), informar la política pública en favor de la equidad y rendir cuentas sobre el compromiso de "no dejar a nadie atrás" $(3,4)$.

En los países de las Américas —una región profundamente desigual- se viene documentando desde hace algunos años con creciente interés la magnitud y las tendencias de las desigualdades sociales en salud - especialmente en el campo de la salud materna e infantil-, tanto a partir de datos administrativos como de encuestas (5-12). Los análisis realizados han permitido, por un lado, fortalecer las capacidades institucionales para la medición y el monitoreo de las desigualdades sociales en salud (13) y, por otro, identificar territorios y grupos poblacionales específicos en situación de mayor vulnerabilidad, donde las desigualdades en salud se concentran desproporcionadamente, y son estos quienes corren el riesgo de "ser dejados atrás".

En este artículo se presenta una metodología para la formulación simultánea de metas cuantitativas que reflejen tanto la mejoría del promedio nacional de un indicador de salud del ODS3 (meta promedial) como la reducción de su desigualdad geográfica (meta distribucional).

\section{MATERIALES Y MÉTODOS}

\section{Datos}

La metodología demanda la disponibilidad de datos sobre indicadores de salud del ODS3 desagregados geográficamente a nivel subnacional (por ejemplo, por estados, departamentos, provincias, cantones, municipios, distritos, comunas o colonias) para un período dado. Esos datos pueden provenir de encuestas de hogares o de registros administrativos. El nivel de desagregación geográfica (i.e., por unidades territoriales) depende de la disponibilidad de datos y su consistencia temporal.

\section{Metodología}

La metodología consiste en un algoritmo de cinco pasos, denominado algoritmo de obtención de metas del ODS3 (AOM_ODS3), explicado a continuación (cuadro 1).

\section{Formulación de la meta promedial}

Si se tiene el valor de un indicador de salud (IS) en dos tiempos definidos, $t_{0} \mathrm{y} t_{1}$, es posible calcular su cambio porcentual anual promedio (CPAP) mediante la siguiente expresión:

$$
\mathrm{CPAP}=\frac{\ln \left(\mathrm{IS}_{t_{1}}\right)-\ln \left(\mathrm{IS}_{t_{0}}\right)}{\left(t_{1}-t_{0}\right)} \times 100
$$

El valor obtenido a partir de la ecuación [1] refleja la velocidad de cambio de un indicador de salud en el tiempo. Si el indicador de salud tiene polaridad negativa (esto es, cuando un valor más bajo del indicador de salud en el transcurso del tiempo

CUADRO 1. Algoritmo de obtención de metas del Objetivo de Desarrollo Sostenible 3 (AOM_ODS3)

Paso 1: Calcular el cambio porcentual anual promedio (CPAP) nacional de un indicador del ODS3 a partir de una serie temporal conocida, con un valor basal y un valor anual de referencia ${ }^{a}$

Paso 2: Definir los estratos geográficos; para ello:

$\checkmark$ Ordenar los valores geográficos del indicador de salud en el tiempo basal según su polaridad (de mayor a menor si tiene polaridad negativa; de menor a mayor si tiene polaridad positiva)

$\checkmark$ Identificar los puntos de corte que definen los estratos geográficos, ya sea por categorías preestablecidas (e.g., por encima y por debajo de un valor referencial nacional establecido) o por grupos según cuantiles (quintiles, cuartiles o terciles).

$\checkmark$ Calcular el promedio ponderado del indicador de salud para cada estrato así definido.

Paso 3: Aplicar el criterio de progresividad proporcional del CPAP a cada estrato definido; para ello:

$\checkmark$ Si el indicador de salud tiene polaridad negativa, asignar un CPAP proporcionalmente más alto cuanto más alto sea el indicador de salud del estrato, según el factor de proporcionalidad.

$\checkmark$ Si el indicador de salud tiene polaridad positiva, asignar un CPAP proporcionalmente más alto cuanto más bajo sea el indicador de salud del estrato, según el factor de proporcionalidad.

$\checkmark$ En cualquier caso, asegurarse de que el promedio aritmético de los CPAP de todos los estratos sea igual al CPAP nacional promedio en el tiempo basal aplicado en el paso 1.

Paso 4: Definir las metas promediales a nivel subnacional y nacional; para ello:

$\checkmark$ Calcular el valor del indicador de salud en un tiempo futuro para cada unidad territorial (meta promedial subnacional) ${ }^{\mathrm{b}}$

$\checkmark$ Calcular el promedio ponderado de los valores del indicador de salud en todas las unidades territoriales en un tiempo futuro (meta promedial nacional).

$\checkmark$ Estos resultados representan las metas a niveles subnacional y nacional del indicador ODS3 en términos absolutos. Al aplicar la expresión [3] ${ }^{c}$ se obtienen las metas en términos relativos.

Paso 5: Definir las metas de reducción de las brechas de desigualdad geográfica; para ello:

$\checkmark$ Calcular las brechas absolutas (BA) y relativas (BR) de desigualdad en los tiempos basal y futuro ${ }^{\text {d }}$.

$\checkmark$ Obtener los cambios porcentuales de la BA y la BR en el períodoc

$\checkmark$ Estos resultados representan las metas de reducción de las brechas de desigualdad geográfica en el indicador ODS3 (metas distribucionales) en términos absolutos y relativos, respectivamente.

Fuente: Elaborado por los autores.

a Ver ecuación [1] en el texto.

Ver la ecuación [2] en el texto.

Ver la ecuación [3] en el texto.

Ver las ecuaciones [4] y [5] en el texto. 
indica una situación más favorable; e.g., tasa de mortalidad), el CPAP mostrará la disminución porcentual anual promedio. $\mathrm{Si}$, por el contrario, el indicador de salud tiene polaridad positiva (esto es, cuando un valor más alto del indicador de salud en el transcurso del tiempo indica una situación más favorable; e.g., cobertura de atención), el CPAP mostrará el aumento porcentual anual promedio.

Asumiendo que se conoce el valor del CPAP y el valor del IS en un tiempo basal, $t_{b}$, en cada unidad geográfica subnacional, se puede establecer una meta subnacional (i.e., para cada unidad geográfica) del IS en un tiempo futuro, $t_{f}$, mediante la siguiente expresión (donde exp es la función exponencial):

$$
\mathrm{IS}_{t_{\mathrm{f}}}=\mathrm{IS}_{t_{\mathrm{b}}} \times \exp \left[\left(\frac{\mathrm{CPAP}}{100}\right) \times\left(t_{\mathrm{f}}-t_{\mathrm{b}}\right)\right]
$$

La meta promedial nacional se define como el promedio ponderado de los valores del indicador de salud en todas las unidades territoriales en el tiempo futuro $t_{f}$. Este promedio ponderado corresponde a una meta nacional expresada en términos absolutos, es decir, retiene las unidades del indicador de salud. Para expresar esta meta en términos relativos - como porcentaje del valor en el tiempo basal- se hace uso de la expresión para el cambio porcentual (CP) del indicador entre el tiempo basal, $t_{b}$, y el tiempo futuro, $t_{f}$, como sigue:

$$
\mathrm{CP}=\frac{\mathrm{IS}_{t_{\mathrm{f}}}-\mathrm{IS}_{t_{\mathrm{b}}}}{\mathrm{IS}_{t_{b}}} \times 100
$$

\section{Formulación de la meta distribucional}

Las metas de reducción de la desigualdad geográfica en salud se expresan cuantitativamente mediante dos métricas estándar: la brecha absoluta y la brecha relativa de desigualdad (BA y $\mathrm{BR}$, respectivamente), que se obtienen a partir de la distribución subnacional definida del indicador de salud para el año meta, abreviándola en estratos geográficos según un criterio de progresividad proporcional del CPAP estrato-específico.

La BA y la BR son medidas-resumen simples de desigualdad en salud y corresponden a la diferencia y al cociente aritméticos, respectivamente, en el valor del indicador de salud entre dos grupos extremos de estratificación geográfica $(3,13)$. Si se cuenta, por ejemplo, con cuatro estratos geográficos ordenados por algún indicador de salud, las métricas de la brecha de desigualdad geográfica se calculan según las siguientes expresiones:

$$
\begin{aligned}
& \mathrm{BA}=\mathrm{IS}_{\mathrm{q} 1}-\mathrm{IS}_{\mathrm{q} 4} \\
& \mathrm{BR}=\frac{\mathrm{IS}_{\mathrm{q} 1}}{\mathrm{IS}_{\mathrm{q} 4}}
\end{aligned}
$$

donde, $q_{1}$ es el primer estrato (que corresponde al estrato con el IS en peor situación) y $q_{4}$ es el cuarto estrato (que corresponde al estrato con el IS en mejor situación).

La BA se expresa en las mismas unidades de medida del indicador de salud; $\mathrm{BA}=0$ denota ausencia de desigualdad. La BR se expresa sin unidades de medida, ya que su valor representa el número de veces que el numerador queda contenido en el denominador; $\mathrm{BR}=1$ denota ausencia de desigualdad $(3,13)$.
A partir de la distribución subnacional ordenada según la situación del indicador de salud (por regla heurística, de la peor a la mejor situación), el AOM_ODS3 propone abreviarla en estratos geográficos (en dependencia del número de unidades territoriales de nivel subnacional se recomienda construir dos, tres, cuatro o, como máximo, cinco estratos), aplicar sobre ellos un criterio de progresividad proporcional en su tasa de CPAP, proyectar sus valores al 2030 según dichas CPAP y computar las brechas absoluta y relativa de desigualdad al 2030. La definición de los estratos se basa en criterios normativos en función del CPAP nacional observado para el indicador de salud del ODS3.

El criterio de progresividad proporcional refleja la aplicación práctica de dos principios básicos relacionados: la ley de rendimiento decreciente o utilidad marginal (la mejoría futura de un indicador de salud es más parsimoniosa cuanto mejor sea su situación actual) (14) y el principio de equidad vertical (la contribución a la mejoría de un indicador de salud debe ser mayor cuanto mayor sea su margen de mejora-mayor necesidad) (15). Desde el punto de vista normativo, para operacionalizar el criterio de progresividad proporcional se fija el factor de progresividad en $50 \%$. En el caso de que se construyan dos estratos $\left(q_{1}\right.$ y $\left.q_{2}\right)$, a $q_{1}$ se le asigna un CPAP 50\% mayor que el CPAP nacional y a $q_{2}$ un CPAP $50 \%$ menor que el CPAP nacional. Si se construyen tres estratos $\left(q_{1}, q_{2}\right.$ y $\left.q_{3}\right)$, se asigna a $q_{1}$ un CPAP $50 \%$ mayor que el CPAP nacional, a $q_{2}$ un CPAP igual al CPAP nacional y a $q_{3}$ un CPAP $50 \%$ menor que el CPAP nacional. Si se construyen cuatro estratos, al estrato con situación de salud más precaria, $q_{1}$, se le asigna un CPAP 50\% mayor que el CPAP nacional y los subsiguientes estratos reciben $+25 \%$, $-25 \%$ y $-50 \%$ del CPAP nacional. En el caso de construir cinco estratos $\left(q_{1}, q_{2}, q_{3}, q_{4}\right.$, y $\left.q_{5}\right)$, se asigna a $q_{1}$ un CPAP $50 \%$ mayor que el CPAP nacional, a $q_{2}$ un CPAP $25 \%$ mayor que el CPAP nacional, a $q_{3}$ un CPAP igual al CPAP nacional, a $q_{4}$ un CPAP $25 \%$ menor que el CPAP nacional y a $q_{5}$ un CPAP $50 \%$ menor que el CPAP nacional. En todos los casos, el promedio de todos los valores de CPAP específicos así definidos para cada estrato satisfacen la propiedad de ser igual al CPAP nacional.

\section{RESULTADOS}

La metodología propuesta se aplicó a los datos administrativos subnacionales de la razón de mortalidad materna (RMM) disponibles para los 22 departamentos de Guatemala en los años 2009 y 2014 (16). A nivel nacional, la RMM fue 140 por 100000 nacidos vivos (nv) en 2009 y 113 por 100000 nv en 2014. Al aplicar la ecuación [1], el CPAP nacional resultante en el período es:

$$
\mathrm{CPAP}=\frac{\ln (113)-\ln (140)}{(2014-2009)} \times 100=-4,3 \%
$$

A partir de los valores de la RMM por departamentos de Guatemala en 2014 ordenados de mayor a menor (cuadro 2), se definieron normativamente cuatro estratos geográficos según dos criterios: un valor referencial nacional de la RMM al 2030 calculado a partir del CPAP nacional $(-4,3 \%)$ y de la RMM nacional basal derivada de la expresión [2] (57 por 100000 nv), y un umbral máximo, equivalente al doble de la meta global del ODS3 para este indicador $(70 \times 2=140$ por 100000 nv). Así, los cuatro estratos agrupan a los departamentos con RMM por encima del umbral máximo (estrato 1), por debajo de este, pero 
CUADRO 2. Valores de la razón de mortalidad materna (RMM) en 2014 y metas proyectadas al 2030 en los departamentos de Guatemala

\begin{tabular}{cccc} 
Estrato & Departamentos & RMM en 2014 & RMM proyectada al 2030 \\
Estrato 1 & Huehuetenango & 232,6 & 88,7 \\
& Totonicapán & 167,7 & 63,9 \\
& Quiche & 162,0 & 61,8 \\
\hline Estrato 2 & Petén & 149,7 & 57,1 \\
& Sacatepequez & 138,5 & 62,0 \\
& Izabal & 131,8 & 59,0 \\
& Chiquimula & 130,6 & 58,5 \\
& Chimaltenango & 129,2 & 57,9 \\
& San Marcos & 127,8 & 57,2 \\
\hline Estrato 3 & Alta Verapaz & 123,9 & 55,5 \\
& Jalapa & 114,0 & 51,0 \\
\hline Sololá & 97,9 & 60,5 \\
& Baja Verapaz & 97,9 & 60,5 \\
& Quetzaltenango & 85,0 & 52,5 \\
& Jutiapa & 74,3 & 45,9 \\
\hline Estrato 4 & Santa Rosa & 71,9 & 44,4 \\
& Escuintla & 65,3 & 40,3 \\
& Suchitepequez & 62,1 & 38,3 \\
& Retalhuleu & 59,5 & 36,7 \\
\hline & Guatemala & 48,0 & 34,8 \\
& Zacapa & 31,6 & 22,9 \\
& El Progreso & 23,4 & 17,0 \\
\hline
\end{tabular}

Fuente: Elaborado por los autores.

por encima del doble del valor referencial nacional (estrato 2), por debajo del doble del valor referencial nacional, pero por encima de él (estrato 3) y por debajo del valor referencial nacional (estrato 4).

A los cuatro estratos así definidos se les asignó un valor de CPAP con progresividad proporcional, a partir del CPAP promedio basal $(-4,3 \%)$ y un factor de progresividad prefijado en $50 \%$. Con estos valores de CPAP específicos para cada estrato se proyectó el valor de la RMM al 2030 para cada departamento del país, es decir, la meta promedial departamental (cuadro 2). A partir de esta nueva distribución departamental de la RMM al 2030 se calculó el valor de la RMM nacional, expresado como promedio ponderado por el tamaño de la población de nacidos vivos en cada departamento (proyección de nacidos vivos al 2030); este promedio ponderado representa en términos absolutos la meta promedial nacional, que en este caso es igual a 53,3 muertes maternas por 100000 nv al 2030. En términos relativos, la meta nacional se expresa como una reducción del 53\% de la RMM nacional entre el 2014 y el 2030. Por otro lado, la distribución departamental de la RMM al 2030 permitió calcular el valor correspondiente a cada estrato, expresado como promedio ponderado por el tamaño de la población de nacidos vivos de cada estrato (proyectada al 2030). En el cuadro 3 se presenta la distribución abreviada de la RMM basal y en el año meta en los cuatro estratos de Guatemala, según los criterios de estratificación y la progresividad proporcional asignada al CPAP por estrato.

Finalmente, con los valores de la RMM basal y al año meta correspondientes a los estratos 1 y 4 (es decir, los estratos extremos de la distribución abreviada: 200,2 y 45,4 muertes
CUADRO 3. Cambio porcentual anual promedio calculado a partir de la razón de mortalidad materna (RMM) a registrada en 2014 y valor proyectado al 2030, en los departamentos de Guatemala, según su estrato de RMM

\begin{tabular}{|c|c|c|c|}
\hline \multirow[t]{2}{*}{ Estratob $^{b}$} & \multicolumn{2}{|c|}{ RMM } & \multirow{2}{*}{$\begin{array}{l}\text { Cambio porcentual } \\
\text { anual promedio }\end{array}$} \\
\hline & 2014 & 2030 & \\
\hline $\begin{array}{l}\text { Estrato } 1 \text { (departamentos } \\
\text { con RMM } \geq 140 \text { ) }\end{array}$ & 200,2 & 76,4 & $-6,4$ \\
\hline $\begin{array}{l}\text { Estrato } 2 \text { (departamentos con } \\
\text { RMM entre } 114,1 \text { y } 140 \text { ) }\end{array}$ & 127,0 & 56,9 & $-5,4$ \\
\hline $\begin{array}{l}\text { Estrato } 3 \text { (departamentos } \\
\text { con RMM entre } 57 \text { y } 114 \text { ) }\end{array}$ & 76,1 & 47,0 & $-3,2$ \\
\hline $\begin{array}{l}\text { Estrato } 4 \text { (departamentos } \\
\text { con } \mathrm{RMM}<57 \text { ) }\end{array}$ & 45,4 & 32,9 & $-2,1$ \\
\hline
\end{tabular}

CUADRO 4. Valores basales y metas promediales y distribucionales de la razón de mortalidad materna (RMM)a al 2030 en Guatemala

\begin{tabular}{lrr}
\multicolumn{1}{c}{ Métrica-resumen de la RMM } & 2014 & 2030 \\
Promedio nacional & 113,0 & 53,3 \\
Brecha absoluta de desigualdad & 154,8 & 43,4 \\
Brecha relativa de desigualdad & 4,4 & 2,3 \\
\hline Fuente: Elaborado por los autores. & &
\end{tabular}

maternas por $100000 \mathrm{nv}$ en el año base, respectivamente, y 76,4 y 32,9 muertes maternas por $100000 \mathrm{nv}$ en el 2030, respectivamente), se calcularon las brechas de desigualdad (BA y BR) en estos dos tiempos, como se muestra en el cuadro 4. Junto con la meta de reducción del promedio nacional, los valores de ambas brechas estimados al 2030 a partir de las ecuaciones [4] y [5] fueron 43,4 y 2,3, respectivamente, y representan las metas distribucionales de reducción de la desigualdad en la RMM para el país (cuadro 4). Si se mantiene la intensidad de reducción de la RMM observada en el país entre los años 2009 y 2014 y si se focalizan sus acciones con progresividad territorial, Guatemala podría reducir en 53\% su RMM nacional, en $72 \%$ su BA de desigualdad geográfica y en $48 \%$ su BR de desigualdad geográfica al 2030 .

\section{DISCUSIÓN}

La metodología presentada en este artículo ha mostrado ser intuitiva, robusta y práctica para hacer explícito el compromiso de "no dejar a nadie atrás" en los esfuerzos nacionales y regionales hacia el cumplimiento de la Agenda 2030 y, más específicamente, el alcance del ODS3 ya mencionado: "garantizar una vida sana y promover el bienestar para todos en todas las edades".

Además de la proverbial meta de mejorar el promedio general de los indicadores, la formulación de metas cuantitativas de reducción de la desigualdad es el primer paso para fortalecer la rendición de cuentas y —mediante su monitoreo- informar las decisiones, las políticas y las intervenciones orientadas al alcance del ODS3 con equidad. De hecho, la metodología presentada aquí adapta al contexto actual un enfoque propuesto 
FIGURA 1. Marco esquemático para el monitoreo del alcance progresivo del Objetivo de Desarrollo Sostenible 3 con equidad

Brecha de desigualdad en salud (2015-2030)

\begin{tabular}{|c|c|c|c|}
\hline & & Estrechándose & Ensanchándose \\
\hline \multirow{2}{*}{ 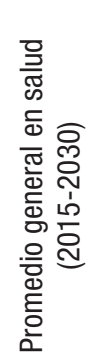 } & Mejorando & $\begin{array}{l}\text { Mejor salud } \\
\text { sin dejar a nadie } \\
\text { atrás }\end{array}$ & $\begin{array}{l}\text { Mejor salud } \\
\text { dejando a más personas } \\
\text { atrás }\end{array}$ \\
\hline & Empeorando & $\begin{array}{l}\text { Peor salud } \\
\text { sin dejar a nadie } \\
\text { atrás }\end{array}$ & $\begin{array}{l}\text { Peor salud } \\
\text { dejando a más personas } \\
\text { atrás }\end{array}$ \\
\hline
\end{tabular}

Fuente: Elaborado por los autores, en base a Minujin y Delamónica (17) y Mújica (18).

originalmente por el Fondo de las Naciones Unidas para la Infancia (UNICEF) en 2002 y lo operacionaliza en un marco de monitoreo esquematizado en la figura $1(17,18)$. Bajo este marco, es fundamental monitorear simultáneamente el promedio territorial de un indicador y la desigualdad de dicho indicador en ese territorio: solamente la mejoría del promedio territorial junto con la reducción de la brecha de desigualdad pueden llevar al escenario esperado de mejorar la salud poblacional sin dejar a nadie atrás.

En sentido general, aunque la declaración de objetivos a los que se aspira en favor de la equidad en la salud es ubicua, la práctica de formular metas explícitas de reducción de desigualdades en salud no se ha consolidado aún en los procesos de planificación sanitaria de todas las regiones, como lo está en países de Europa occidental, especialmente en el Reino Unido (19). En las Américas, los esfuerzos más visibles al respecto los ha catalizado la Organización Panamericana de la Salud (20-22), muy notablemente en sus dos últimos planes estratégicos sexenales $(23,24)$, en los que los Estados Miembros aprobaron metas explícitas de reducción de las desigualdades sociales en salud, en consonancia con el primer objetivo de su misión: promover la equidad en la salud. El advenimiento de la Agenda 2030, con sus ODS, y las lecciones aprendidas en la implementación de la Declaración del Milenio, con sus ODM, (25) han renovado el sentido de urgencia de poner en práctica sistemas de monitoreo de las desigualdades sociales, incluidas las de la salud $(26,27)$. En el reciente Informe Final de la Comisión sobre Equidad y Desigualdades en Salud en las Américas, liderada por Sir Michael Marmot, se destaca en su Recomendación 11.A: "hacer que la equidad en la salud sea un indicador clave del desarrollo de la sociedad y establecer mecanismos de rendición de cuentas", y se reconoce el monitoreo de desigualdades en salud como un mecanismo prioritario de gobernanza para lograr la equidad en la salud (28).

El AOM_ODS3 pretende servir solamente como una guía práctica y flexible para promover acciones institucionales específicas a partir de la cuantificación de los cambios deseados y factibles en la distribución de la salud y el bienestar, e informar las decisiones orientadas a cumplir el ODS3. La flexibilidad inherente a este algoritmo atañe, al menos, a tres aspectos centrales: a) el nivel de desagregación de los datos, pues el algoritmo puede servir tanto al nivel local — como los barrios de una ciudad - como al nivel regional — países de un continente-; b) el criterio de estratificación de las unidades territoriales, es decir, la selección de los puntos de corte de la distribución basal del indicador de salud, para lo cual existen alternativas metodológicas a la aquí presentada; y c) los criterios de progresividad proporcional aplicados a la velocidad del cambio proyectado - es decir, el CPAP - del indicador de salud al 2030, que también tiene múltiples opciones metodológicas y refleja, esencialmente, el grado de aversión a la desigualdad que exhibe cada sociedad (29.) Estos tres aspectos centrales, entre otros, pueden considerarse como oportunidades directas para el diálogo y el consenso interdisciplinario e intersectorial en los niveles correspondientes, necesarios para implementar esta propuesta. La formulación de metas de reducción de desigualdades en la salud no debe verse como un ejercicio matemático-estadístico, sino como un trabajo colaborativo de construcción colectiva en favor de la equidad. En el caso práctico presentado, por ejemplo, la aplicación del AOM_ODS3 podría llevar a Guatemala a implementar políticas dialogadas y consensuadas que permitan no solamente reducir a la mitad la RMM en el año 2030 (en este caso de 113 por 100000 nv a 53 por 100000 nv), sino — simultáneamente - reducir drásticamente la desigualdad geográfica subnacional en el riesgo de muerte materna: reducir tres cuartas partes la brecha absoluta (es decir, de 155 por $100000 \mathrm{nv}$ a 44 por $100000 \mathrm{nv}$ ) y a la mitad la brecha relativa (es decir, de 4,4 a 2,3), con el consiguiente beneficio sanitario y social que ello conlleva.

La aplicación efectiva del algoritmo es tributaria de la calidad de los datos disponibles. Ello podría representar una limitación, especialmente con el uso de datos administrativos subnacionalmente desagregados, si los datos crudos no son sometidos previamente a procedimientos estándar (30) de crítica y control de la calidad (e.g., subregistro en las estadísticas vitales, mala clasificación de las muertes, inestabilidad de los números pequeños, entre otros). Como en todo trabajo analítico, en los datos empleados para la formulación de las metas de reducción de la desigualdad en salud se recomienda preservar los principios de base poblacional, la deconfusión y la precisión comparable (31). Otra limitación del algoritmo propuesto puede surgir de la presencia del sesgo residual inherente al diseño ecológico, especialmente cuando se emplean datos de fuentes administrativas, por lo que se recomienda utilizar datos con la mayor desagregación geoespacial posible. 
No obstante esas limitaciones, la metodología propuesta ofrece un correlato territorial directo en cuanto al comportamiento de los indicadores del ODS3, a la vez que permite identificar las unidades territoriales que concentran las mayores desigualdades en la salud y que, en consecuencia, corren el mayor riesgo de "quedarse atrás". Con esto, se brinda una valiosa información para el establecimiento de metas territoriales, la toma de decisiones y la implementación de estrategias e intervenciones apropiadamente focalizadas.

El sentido de urgencia para enfrentar y eliminar las desigualdades injustas en las oportunidades para la salud y el bienestar en el camino hacia la salud universal y el desarrollo sostenible que ha sido mundialmente acordado en la Agenda 2030, se ha visto súbita y dramáticamente avivado por la irrupción de la pandemia del nuevo coronavirus SARS-CoV-2 y la COVID19, que ha expuesto y amplificado las desigualdades sociales y, sobre todo, las desigualdades en salud (32). El camino hacia el cumplimiento de las metas del 2030 tiene ahora características pospandémicas y, en consecuencia, la sociedad en su conjunto ha de revisar y replantear sus prioridades. En ese proceso, se debe reafirmar la primacía del principio de equidad en la salud para el alcance del ODS3, si renueva su promesa de no dejar a nadie atrás.

Las decisiones y las acciones en favor del cumplimiento del ODS3, sobre todo bajo el escenario pospandémico, se verán mejor informadas si se guían por metas explícitas en mejorar el promedio nacional de los indicadores del ODS3 y en la reducción de sus desigualdades.
Contribución de los autores. AS (https://orcid.org/00000002-1857-3662), IE (https://orcid.org/0000-0002-0304-0492), OJM (https:/ / orcid.org/0000-0002-2893-4206) y JBS (https:/ / orcid.org/0000-0002-3078-9642) concibieron el estudio original, analizaron los datos, y escribieron y revisaron el manuscrito. Todos los autores revisaron y aprobaron la versión final.

Agradecimientos. Los autores dejan constancia de su reconocimiento a la iniciativa Todas las Mujeres Todos los Niños en Latinoamérica y el Caribe (EWEC-LAC, por sus siglas en inglés) y al equipo de asesores regionales del Departamento de Evidencia e Inteligencia para la Acción en Salud (EIH) de la Organización Panamericana de la Salud/Organización Mundial de la Salud por el constante apoyo al desarrollo de la propuesta presentada.

Conflictos de intereses. Los autores declaran no tener conflictos de intereses.

Financiación. Este estudio no ha recibido financiación.

Declaración. Las opiniones expresadas en este artículo son únicamente responsabilidad de los autores y no reflejan necesariamente los criterios ni la política de la Revista Panamericana de Salud Pública / Pan American Journal of Public Health y/o la Organización Panamericana de la Salud.

\section{REFERENCIAS}

1. United Nations General Assembly. Transforming our World: the 2030 Agenda for Sustainable Development (A/ RES/70/1). New York: UN; 2015 [citado el 19 de agosto de 2020]. Disponible en: https://www.un.org/en/development/desa/population/migration/generalassembly/docs/globalcompact/A_RES_70_1_E.pdf

2. United Nations Statistics Division. Global indicator framework for the Sustainable Development Goals and targets of the 2030 Agenda for Sustainable Development (A/RES/70/1; E/CN.3/2018/2). New York: UN; 2018 [citado el 19 de agosto de 2020]. Disponible en: https://unstats.un.org/sdgs/indicators/Global\%20Indicator\%20 Framework\%20after\%202020\%20review_Eng.pdf

3. Mújica OJ, Moreno CM. De la retórica a la acción: medir desigualdades en salud para "no dejar a nadie atrás". Rev Panam Salud Publica. 2019;43:e12.

4. Fukuda-Parr, S. Keeping out extreme inequality from the SDG Agenda - the politics of indicators. Glob Policy. 2019;10(Suppl.1):61-9.

5. Organização Pan Americana da Saúde. Atlas de desenvolvimento sustentável e saúde: Brasil 1991-2010. Brasilia: OPAS; 2015.

6. United Nations Children's Fund, Tulane University. Health Equity Report 2016: analysis of reproductive, maternal, newborn, child, and adolescent health inequities in Latin America and the Caribbean to inform policymaking. New York: UNICEF; 2016 [citado el 19 de agosto de 2020]. Disponible en: https:/ / www.everywomaneverychild-lac.org/e/publication/health-equity-report-2016/

7. Sanhueza A, Calle-Roldán J, Ríos-Quituizaca P, Acuña MC, Espinosa I. Social inequalities in maternal mortality among the provinces of Ecuador. Rev Panam Salud Publica. 2017;41:e97.

8. Casalino-Rojo E, Ochoa-Amenábar E, Mújica OJ, Munayco CV. Desigualdades sociogeográficas en la mortalidad materna en Perú: 2001-2015. Rev Peru Med Exp Salud Publica. 2018;35(2):351-3.

9. Mesenburg MA, Restrepo-Méndez MC, Amigo H, Balandrán AD, Barbosa-Verdún MA, Caicedo-Velásquez B, et al. Ethnic group inequalities in coverage with reproductive, maternal, and child health interventions: cross-sectional analyses of national surveys in
16 Latin American and Caribbean countries. Lancet Glob Health. 2018;6:e902-13.

10. Cecon R, Meneghel S, de Menezes V, Bueno A, Arguedas G, Hahn A. Maternal mortality in the provincial capitals of Brazil. Rev Cub Salud Publica. 2019;45(3):e835.

11. Enríquez-Nava MF, Esquivel-Velásquez AD, Patón-Sanjines $M$, Pooley-Ayarza BC, Alarcón R, Hernández-Muñoz R, et al. Comportamiento y desigualdades sociales en indicadores priorizados del Objetivo de Desarrollo Sostenible 3 en Bolivia. Rev Panam Salud Publica. 2020;44:e101.

12. Tullo E, Lerea MJ, González R, Galeano J, Insfrán MD, Muñoz M, et al. Desigualdades sanitarias y sociales en la salud materna y del niño en Paraguay. Rev Panam Salud Publica. 2020;44:e107.

13. Organización Panamericana de la Salud. Manual para el monitoreo de las desigualdades en salud, con especial énfasis en países de ingresos medianos y bajos. Washington, DC: OPS; 2016 [citado el 19 de agosto de 2020]. Disponible en: https://www.paho.org/ hq/dmdocuments/2016/manual-moni-desig-sociales-salud-2016 .pdf

14. Brue SL. The law of diminishing returns. J Econ Perspect. 1993;7(3):185-92.

15. Kakwani NC, Wagstaff A, van Doorslaer E. Socioeconomic inequalities in health: measurement, computation and statistical inference. J Econom. 1997;77(1):87-103.

16. Iniciativa Todas las Mujeres Todos los Niños en Latinoamérica y el Caribe. Perfiles de desigualdades en salud materna, infantil y adolescente: Guatemala. Washington, DC: EWEC-LAC; 2017 [citado el 19 de agosto de 2020]. Disponible en: https://www. everywomaneverychild-lac.org/wp-content/uploads/2019/08/ perfil_guatemala_17abril.pdf

17. Minujin A, Delamónica E. Mind the gap! Widening child mortality disparities. J Hum Dev. 2003;4(3):397-418.

18. Mújica OJ. Trascendiendo la invisibilidad: el monitoreo de las desigualdades sociales en salud. Hacia Promoc Salud. 2016;21(1):9-11. 
19. Crombie IK, Irvine L, Elliot L, Wallace H. Closing the health inequalities gap: an international perspective. Copenhagen: World Health Organization; 2005 [citado el 19 de agosto de 2020]. Disponible en: https:// www.euro.who.int/_data/assets/pdf_file/0005/124529/E87934.pdf

20. Etienne CF. Achieving social equity. In: Frenk J, Hoffman SJ, eds. To Save Humanity: what matters most for a healthy future. New York: Oxford University Press; 2015.

21. Becerra-Posada F. Equidad en salud: mandato esencial para el desarrollo sostenible. Rev Panam Salud Publica. 2015;38(1):1-4.

22. Choi BCK, Maza RA, Mújica OJ, PAHO Strategic Plan Advisory Group, PAHO Technical Team. The Pan American Health Organization-adapted Hanlon method for prioritization of health programs. Pan Am J Public Health. 2019;43:e61.

23. Organización Panamericana de la Salud. Plan Estratégico de la OPS 2014-2019. En pro de la salud: desarrollo sostenible y equidad. Washington, DC: OPS; 2014 [citado el 19 de agosto de 2020]. Disponible en: https: / /iris.paho.org/handle/10665.2/4034

24. Organización Panamericana de la Salud. Agenda de Salud Sostenible para las Américas 2018-2030. Un llamado a la acción para la salud y el bienestar en la Región. Washington, DC: OPS; 2017 [citado el 19 de agosto de 2020]. Disponible en: https://iris.paho.org/ handle/10665.2/49169

25. Organización Panamericana de la Salud. Salud en las Américas, edición 2017. Resumen: panorama regional y perfiles de país. Washington, DC: OPS; 2017 [citado el 19 de agosto de 2020]. Disponible en: https://iris.paho.org/handle/10665.2/34322

26. Hosseinpoor AR, Bergen N, Magar V. Monitoring inequality: an emerging priority for health post-2015. Bull World Health Organ. 2015;93:591-A.
27. Hosseinpoor AR, Bergen N, Schlotheuber A, Grove J. Measuring health inequalities in the context of sustainable development goals. Bull World Health Organ. 2018;96:654-9.

28. Comisión de la Organización Panamericana de la Salud sobre Equidad y Desigualdades en Salud en las Américas. Sociedades justas: equidad en la salud y vida digna. Informe de la Comisión de la Organización Panamericana de la Salud sobre Equidad y Desigualdades en Salud en las Américas. Washington, DC: OPS; 2019 [citado el 19 de agosto de 2020]. Disponible en: https://iris.paho .org/handle/10665.2/49505

29. Wagstaff A. Inequality aversion, health inequalities and health achievement. J Health Econ. 2002;21:627-41.

30. Murray CJL. Towards good practice for health statistics: lessons from the Millennium Development Goal health indicators. Lancet. 2007;369:862-73.

31. Wacholder S, McLaughlin JK, Silverman DT, Mandel JS. Selection of controls in case-control studies: I. Principles. Am J Epidemiol. 1992;135(9):1019-28.

32. Marmot M, Allen J. COVID-19: exposing and amplifying inequalities. J Epidemiol Community Health. 2020;74(9):681-2.

Manuscrito recibido el 20 de agosto del 2020. Aceptado para publicación, tras revisión, el 1 de octubre del 2020. 


\section{Leaving no one behind: Methodology to set health inequality reduction targets for Sustainable Development Goal 3}

ABSTRACT Objectives. Present methodology for the concurrent development of quantitative targets that reflect improvement in the national average of an indicator for Sustainable Development Goal 3 (SDG3), as well as a reduction in geographic inequality.

Methods. A five-step algorithm was developed: a) calculate the national average annual percentage change (AAPC) for an SDG3 indicator; b) standardize the definition of geographic strata based on subnational distribution of the indicator in a base year; c) apply a criterion for proportional progress in the AAPC in order to project the stratum-specific indicator to the target year; d) set the national target as the weighted average of the indicator in the subnational territorial units for the target year; and e) develop inequality reduction targets by calculating absolute and relative gaps between the top and bottom strata for the target year.

Results. The algorithm was applied to SDG indicator 3.1.1 (maternal mortality ratio, MMR), disaggregated by Guatemala's 22 departments for base year 2014 (MMR = 113/100,000 live births). By sustaining the average AAPC rate attained from 2009 to $2014(-4.3 \%)$ and targeting its actions to territorial progress, the country would reduce its MMR to 53/100,000 by 2030 and its absolute and relative gaps by $72 \%$ and 48\%, respectively.

Conclusions. The proposed methodology makes it possible to concurrently develop targets for the reduction of geographic inequalities in health and improvements in the national average, with explicit reference to the primacy of the principle of equity expressed in the SDGs' commitment to leaving no one behind, whose urgency is newly important in the current post-pandemic scenario.

Keywords Sustainable development; health equity; health status indicators; maternal mortality; Guatemala.

\section{Não deixar ninguém para trás: uma metodologia para estabelecer metas de redução das desigualdades em saúde sob o Objetivo de Desenvolvimento Sustentável 3}

RESUMO

Objetivos. Apresentar uma metodologia para a formulação simultânea de metas quantitativas que reflitam tanto a melhoria da média nacional de um indicador do terceiro Objetivo de Desenvolvimento Sustentável (ODS3) quanto a redução das desigualdades geográfica nesse indicador.

Métodos. Estabelecemos um algoritmo em cinco etapas: (a) cálculo da variação percentual anual média (VPAM) em um país para um indicador do ODS3, (b) definição normativa de estratos geográficos a partir da distribuição subnacional do indicador em um ano base, (c) aplicação de um critério de progressividade proporcional da VPAM para projetar o indicador específico do estrato para o ano base, (d) estabelecimento da meta nacional como a média ponderada do indicador nas unidades territoriais subnacionais para o ano alvo e (e) estabelecimento de metas para a redução das desigualdades calculando a disparidade absoluta e relativa entre os estratos extremos para o ano alvo.

Resultados. Aplicamos o algoritmo ao indicador ODS 3.1.1 (razão de mortalidade materna, RMM), desagregado pelos 22 departamentos da Guatemala para o ano base de 2014 (RMM = 113 por 100.000 nascidos vivos). Se mantiver a intensidade média da VPAM observada entre 2009 e 2014 (-4,3\%) e concentrar as suas ações com progressividade territorial, o país reduzirá, até 2030, a sua RMM para 53 por 100.000 e sua disparidade absoluta e relativa em $72 \%$ e $48 \%$, respectivamente.

Conclusões. A metodologia proposta permite formular simultaneamente metas para a redução das desigualdades geográficas em saúde e explicitar a primazia do princípio da equidade expresso no compromisso de não deixar ninguém para trás consagrado nos ODS, cuja urgência assume uma relevância renovada no atual cenário pós-pandêmico.

Palavras-chave Desenvolvimento sustentável; equidade em saúde; indicadores básicos de saúde; mortalidade materna; Guatemala. 